\title{
Displacement Prediction of Surrounding Rock in Tunnel Based on cellular ant algorithm
}

\author{
LI Yongyu $^{1}$, WANG Yu ${ }^{1,2, *}$, and WANG Shihua ${ }^{1}$ \\ ${ }^{1}$ Shanghai Urban Construction Vocational College, 200438, Shanghai, China \\ ${ }^{2}$ Shanghai Institute of Technology, 201418, Shanghai, China
}

\begin{abstract}
The deformation of tunnel surrounding rock is the key factor to analyze the stability of surrounding rock. However, due to the influence of many factors and the strong non-linear relationship between the factors, it is difficult to predict the deformation effectively. In this paper, a method based on cellular ant neural network model is proposed to simulate the displacement of surrounding rock with time. The results show that this method is efficient and feasible, and can meet the requirements of engineering and control.
\end{abstract}

\section{Overview}

As a special kind of underground engineering, the construction process of tunnel engineering is affected by many uncertain factors. In order to ensure the safety of construction, it is particularly important to monitor the deformation displacement of tunnel surrounding rock in the process of excavation. By monitoring the change of displacement, the stability can be controlled according to the needs. Through long-term data analysis, we can find out the time series relationship of surrounding rock deformation. This relationship reflects strong non-linear, fuzzy and strong noise. Ant algorithm is a kind of evolutionary algorithm. It has a good ability of searching for the optimal combination problem. This algorithm imitates the cooperation between ants: biologists have proved that the communication between ants is completed through pheromone, and ants always prefer to choose the path with rich pheromone. Cellular automata (CA) is a network dynamic model with discrete time, space and state, and local interaction and causality in time. Its basic principle is to simulate complex and rich macro phenomena by parallel evolution of a large number of cells under simple rules. Cellular ant algorithm (CA) is a new optimization algorithm formed by adding the idea of cellular automata to ant algorithm. It retains the good searching ability of ant algorithm and further avoids falling into local optimum.

\section{Cellular model ant algorithm}

\section{1 cellular evolution rules}

(1) Select any cell ci and calculate $Z=f(x 1, x 2, \ldots . . x n)$, and record $\mathrm{Zopt}=\mathrm{Z}$, Copt $=\mathrm{Ci}$

(2) Select the region $\mathrm{Ni}$ and $\mathrm{Nj}$ arbitrary cells ci and cj in the neighborhood to calculate $\mathrm{Zi}$ and $\mathrm{Zj}$
When $\mathrm{Zi}<\mathrm{Zj}$ and $\mathrm{Zopt}<\mathrm{Zj}$, the value of information intensity update $\Delta \tau_{\mathrm{jk} \text { increases by } \mathrm{Q}}$

When $\eta_{i j}>0$, ant i moves from its neighborhood i to ant $\mathrm{j}$ 's neighborhood according to the probability $\mathrm{Pij}$; then the region $\mathrm{Ni}$ dies $\mathrm{Ni}$

When $\eta_{i j} \leq 0$, ant $\mathrm{i}$ continues to search in the region

\section{2 pheromone update}

$\tau_{i j}(t+n)=\lambda \tau_{i j}(t)+\Delta \tau_{i j}$ Where $\lambda$ is the parameter, $(1-\lambda)$ represents the evaporation rate of pheromone between time $t$ and $t+n$.

$$
\Delta \tau_{i j}=\sum_{k=1}^{n} \Delta \tau_{i j}^{k}
$$

$\Delta \tau$ ijkIs the number of information left by the kth ant on edge e(i,j) in the unit length between time $\mathrm{t}-\mathrm{t}+\mathrm{n}$.

$\Delta \tau_{\mathrm{ijk}}=-\quad 0$ other

Where $\mathrm{Q}$ is a constant and LK is the distance traveled by the kth ant.

\section{3 neighborhood transfer probability of ants}

The transfer probability of the kth ant from $\mathrm{i}$ position to $\mathrm{j}$ position is as follows.

\footnotetext{
* Corresponding author: wangyu@succ.edu.cn
} 


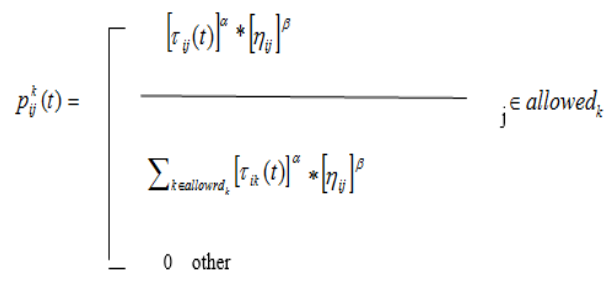

among, allowedk $=\{\mathrm{N}$-tabuk $\}$, aand $\beta$ are parameters that control the relative importance of pheromone and visibility. Transition probability is a trade-off between visibility and pheromone strength at time t. $\eta_{i j}$ is the visibility of the side $\operatorname{arc}(\mathrm{i}, \mathrm{j})$, i.e. 1 / dij.

\section{4 the main steps of cellular ant algorithm are as follows}

(1) nc $\leftarrow 0$; (nc is the number of iteration steps or search times) and $\mathrm{j}$ initialization; the size of the area is determined according to the number of ants $(\mathrm{m})$ and the size of the space, and $\mathrm{M}$ ants are placed in the center of the M search areas; (2) The initial starting point of each ant is placed in the current solution set; for each ant $\mathrm{K}(\mathrm{k}$ $=1, \ldots, \mathrm{m})$, region search is carried out, and the probability pijk moves to the neighborhood of $\mathrm{J}$; then the region $\mathrm{Ni}$ dies; (3) the objective function value $\mathrm{Z}$ of each ant is calculated; the current best solution is recorded; (4) the trajectory strength is modified according to the update process; (5) $\triangle \mathrm{r} \leftarrow 0$; nc $\leftarrow$ nc+1; (6) If the number of iterations is less than a predetermined number of iterations and there is no degenerative behavior (that is, all the solutions found are the same), then turn to step 2; (7) output the best solution at present. With the evolution of life and death of each region, ants will focus on some regions, which will speed up the optimization process

\section{Cellular ant neural network algorithm for tunnel surrounding rock deformation}

The deformation of tunnel surrounding rock is affected by structural load, initial stress state and geotechnical parameters. The following aspects can be considered: initial stress $\mathrm{P}$, cohesive force $\mathrm{C}$, internal friction angle $\varphi$, Poisson's ratio $\mu$, elastic modulus of rock mass $\mathrm{E}$

The following optimization objective functions can be constructed:

$$
\operatorname{minK}(\mathrm{x})=\sum_{i=1}^{n}\left\|\left[K_{i}\left(x, X_{l}\right)-u_{i}\right]^{2}\right\|
$$

Where $\mathrm{x}=[\mathrm{x} 1, \mathrm{x} 2, \ldots \ldots \mathrm{xn}]=[], \mathrm{X}$ is the input vector of the $\mathrm{N}$-dimensional model; $\mathrm{X}$ is the output vector of the
M-dimensional model, ui is the data vector corresponding to the M-dimensional output vector $\mathrm{Xi}$. In the objective function, the number of optimization parameter vectors $n$ is determined according to the number of slices. Cellular ant algorithm can speed up the convergence speed to the greatest extent, greatly improve the speed of network learning and calculation accuracy,

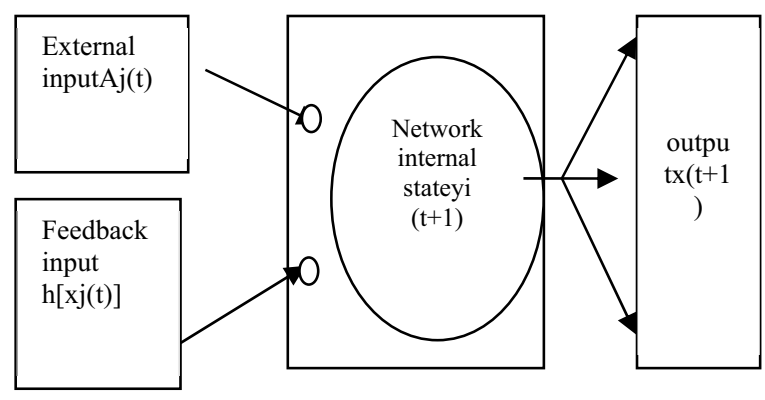

Fig 1. cellular ant neural network model

Each neuron is affected by both external input $\mathrm{Aj}(\mathrm{t})$ and internal feedback $\mathrm{h}[\mathrm{xj}(\mathrm{t})]$ at the same time, and this effect declines with time like the refractory term $g[x i(t)]$. Therefore, each neuron is affected by all the neurons in the network. Therefore, the network is multi input and single output (Fig. 1). After considering the above effects, the cellular ant neural network model can be constructed on the basis of the neuron model:

$$
\begin{aligned}
& x_{i}(t+1)=f\left\{\sum_{j=1}^{M} v_{i j} \sum_{r=0}^{t} k^{r} A_{j}(t-r)+\sum_{j=1}^{N} \omega_{i j} \sum_{r=0}^{t} k^{r} h\left[x_{j}(t-r)\right]-\alpha \sum_{r=0}^{t} k^{r} g\left[x_{i}(t-r)\right]-\theta\right\}
\end{aligned}
$$

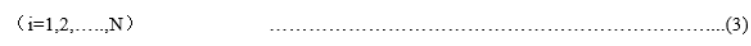

$$
\begin{aligned}
& y_{i}(t+1)=k y_{i}(t)+\sum_{j=1}^{M} v_{i j} A_{j}(t)+\sum_{j=1}^{N} \omega_{i j} h\left\{f\left[y_{j}(t)\right]\right\}-\alpha g\left\{f\left[y_{i}(t)\right]\right\}-\theta_{i}(1-k) \ldots \ldots(4)
\end{aligned}
$$

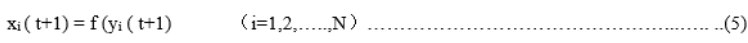

Formula (4) and formula (5) are cellular ant neural network models, which can be used to evaluate and predict the deformation of tunnel surrounding rock. The calculation is based on a tunnel engineering example.

\section{Engineering case analysis}

In this paper, the cellular ant neural network algorithm model is optimized and predicted by using the deformation monitoring data of the right vault (DK17 + 124.85 section) of a tunnel project in a slope section. In this paper, the first 30 groups of collected data are used as training sample data, and three-layer cellular ant neural network (can) is used for learning and testing, and the last 12 groups are the predicted values. The accuracy of the data is evaluated through the test results. The specific calculation and analysis results are shown in Table 1

Table1. monitoring value of DK17 +124.85 section displacement and calculation value of cellular ant algorithm neural network model

\begin{tabular}{|c|c|c|}
\hline Number & Date & Measured displacement/mm Estimate \\
\hline 1 & 20040311 & 0.17 \\
\hline 2 & 20040315 & 0.19 \\
\hline 3 & 20040318 & 0.26 \\
\hline
\end{tabular}




\begin{tabular}{|c|c|c|}
\hline 4 & 20040321 & 0.24 \\
\hline 5 & 20040326 & 0.27 \\
\hline 6 & 20040329 & 0.30 \\
\hline 7 & 20040402 & 0.30 \\
\hline 8 & 20040407 & 0.32 \\
\hline 9 & 20040410 & 0.38 \\
\hline 10 & 20040415 & 0.37 \\
\hline 11 & 20040419 & 0.43 \\
\hline 12 & 20040423 & 0.51 \\
\hline 13 & 20040428 & 0.57 \\
\hline 14 & 20040501 & 0.60 \\
\hline 15 & 20040505 & 0.59 \\
\hline 16 & 20040509 & 0.66 \\
\hline 17 & 20040513 & 0.70 \\
\hline 18 & 20040517 & 0.71 \\
\hline 19 & 20040520 & 0.78 \\
\hline 20 & 20040523 & 0.80 \\
\hline 21 & 20040526 & 0.83 \\
\hline 22 & 20040529 & 0.81 \\
\hline 23 & 20040602 & 0.88 \\
\hline 24 & 20040605 & 0.89 \\
\hline 25 & 20040608 & 0.96 \\
\hline 26 & 20040611 & 1.01 \\
\hline 27 & 20040615 & 1.06 \\
\hline 28 & 20040618 & 1.11 \\
\hline 29 & 20040620 & 1.10 \\
\hline 30 & 20040624 & 1.23 \\
\hline 31 & 20040627 & 1.263 \\
\hline 32 & 20040630 & 1.302 \\
\hline 33 & 20040703 & 1.365 \\
\hline 34 & 20040708 & 1.398 \\
\hline 35 & 20040710 & 1.457 \\
\hline 36 & 20040715 & 1.487 \\
\hline 37 & 20040728 & 1.582 \\
\hline 38 & 20040731 & 1.675 \\
\hline 39 & 20040802 & 1.697 \\
\hline 40 & 20040806 & 1.703 \\
\hline 41 & 20040810 & 1.733 \\
\hline 42 & 20040813 & 1.736 \\
\hline
\end{tabular}

\section{Conclusion}

The time series prediction based on the combination of cellular ant algorithm and neural network is realized by reconstructing the phase space. The engineering example shows that this model can achieve good results in the practical application. It can be seen that in the extrapolation prediction, the error between the predicted value and the measured value is controlled within $2 \%$, which has strong reliability. The prediction model of cellular ant neural network algorithm can guide us to 
evaluate the stability of tunnel structure to a certain extent. Practice shows that with the increase of sample number, the prediction effect and prediction accuracy of the prediction model based on cellular ant neural network will be greatly improved. It can be seen that this method has certain theoretical value and application prospect.

\section{Reference}

1. Wolfram s. Theory and Application of Cellular Automata[M].Singapore: The world Scientific Pubilishing Co, Ltd, 1986

2. Yang Dixiong, Li Gang, Cherng Gengdong. comparative study on chaos optimization algorithm for nonlinear function $[\mathrm{J}]$. Chinese Journal of Computational Mechanics ,2004,21(3);257-262.

3. Feng XiaTing, guide wheel of intelligent rock mechanics [M]. Beijing, Science Press, 2000

4. Ma Liang, ant algorithm for discrete system optimization [D]. Shanghai: Shanghai Jiaotong University, 1999 